\title{
MEMBERSHIP
}

Changes of Address ANd Section -Sept. 1, 1950-Nov. 30, 1950.

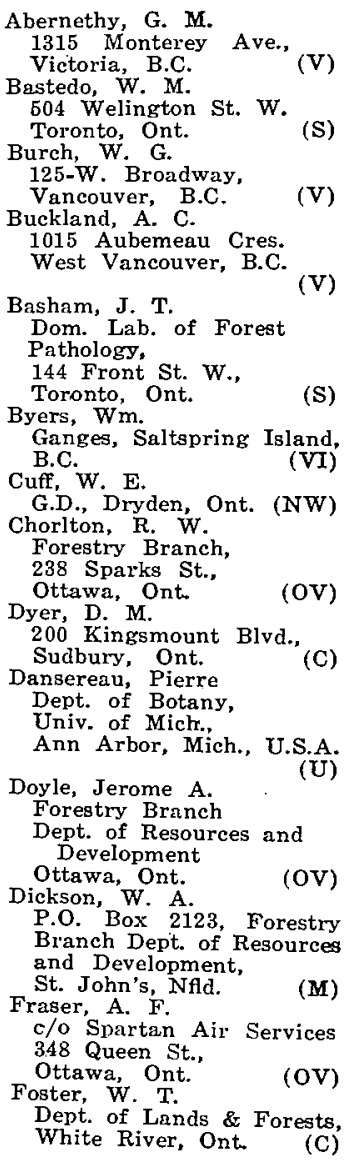

Adamson, M. A. Midhurst, On't.

Adlam, W. D.

Dept. Lands \& Forests, Saint Williams, Ont. (SO)

Balkwill, R. A.

Forest Resources

Inventory,

504 Wellington St. W Toronto

Bothwell, J. E.

139 Church St.,

Sault Ste. Marie, Ont.

Brown, W. G. E

24 Yonge St. N.

Richmond Hill, Ont. (S)
Giles, J. Walter

1771 Fulmar St.

Ann Arbor, Mich (USA)

Gracie, J. E. Abitibi P.\& P. Co.s
Sturgeon Falls, Ont. (C)

Hall, J. R. H. Lambton Apts. 1045-97th St.,

Edmonton, Alta.
Hallett, M. J.

(RM)

516 Adelaide St. Dalhousie, N.B.

Hawboldt, L $S$.

Division of Forest Biology

N.S. Dept. of Lands \&

Forests,

513 Prince St.

Truro, N.S.

(M)

Harrison, W. C.

c/o Manitoba Paper Co

Ltd., Pine Falls.

Man.

Co.

ohnston, E. F.

Dept. of Lands \& Forests,

Miller Lake, Ont.

Koerner, W. C.

401 Marine Bldg.,

Vancouver, B.C.

ittle, L. E. J.

19 Rue Cardinal

Villeneuve,

Chicoutimi, Que.
Lyford, P. L.

Suite 1017 Vancouv

736 Granville St.

LeBlanc, Ien

P.O. Box 901

Fredericton, N.B.

Fredericton,

B. (M) Anglo-Can. Pulp \& Paper Mills,

Quebec, P.Q

Deputy G. P.

Deputy Min. of Lands,

Dept. of Lands \& Forests

Victoria, B.C.

Matheson, C. R.

c/o C. D. Schultz,

Vancouver, B.C.

(V)

(V)

(M)

(VI)

(V)

(V)

New Full Members

Conway, J. M.

Consol Paper Corp. Ltd. St. Michel Des Saints,

Berthier, P.Q.

(Q)

Cox, Graham Ont. Minn. P. \& $\mathrm{P}$ ( Co.,
Kenora, Ont.

Dixon, R. M.

35 Admiral Rd.

Toronto, Ont.

Denyer, W. B. G.

Dom. Lab. of Forest

Pathology,

Univergity of Sask.,

Saskatoon, Sask

Fletcher, G. L.

Dept. Lands \& Forests

Algoma Central Railway,

Sault Ste. Marie, Ont. (C)
MacBean, A. P.

Qualicun Beach, B.C.

McElhanney, T. A.

Box 553,

Nickerson, Ont.

P.O. Box 2123,

Dept. Resources \& Development,

Royce, C. D.

Ryce, C. D.

c/o M. N. Simpson, O.L.S Homestead Block, North Bay, Ont

North Bay, Ont.

Russell, M. D. Woodlands Division

Bathurst P. \& P. Co., Ltd

West Bathurst, N.B. (M)

Slipp, A. W.

School of Forestry Univergity of Idaho Moscow, Idaho

Thomson, T. M.

Kennedy Lake Log. Co.,

Ucluelet, B.

Trorey, L. G. (V) 4827 College Highroad
Vancouver, B.C. (V)

Thomas, L. B. 288 Putney Ave., St. Lambert, Que.

Ternan, C. C. Room 11, Passmore Bldg. 345 Victoria St.,

Whittaker, J. E

c/o Restigouche Co Ltd Campbellton, N.B. (M) Wilson, D. A.

2C, 4200 Stockton St. Richmond, Calif., USA

Wilde, C. J. R Pineland Timber Co.,
Foleyet, Ont.

Yeomans, D. G. Indian Affairg Branch,

Camp Chippewa,

Hummel, R. W.

611 Bay St., Toronto (S) Marathon Paper Mills, Camp 35,

Caramat, Ont. (NW)

Huc, J. L.

4954 St. Catherine East,

Montreal, P.Q.

Ottawa Field Est.

Ottawa Field Est.

Forest Insect Inv.,

Science Service Bldg.

Ottawa, Ont.
Harvie, $P$. $M$.

19 Dundonald St.,

Barrie, Ont.
rause, R. F.

Whitecourt, Alta. 


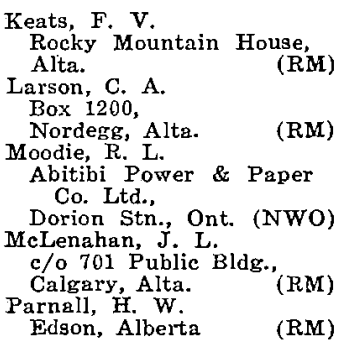

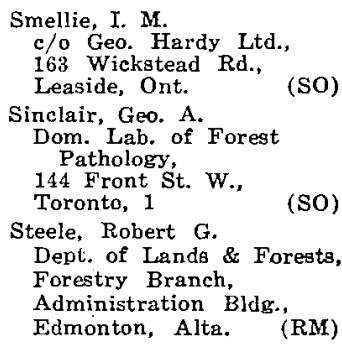

Scott, D. R. M 701 Public Bldg., Calgary, Alta.

(RM)

Wallis, J. R.

c/o Mrs. J. Asser

W. Vancouver, B.C. (V)

Williams, J. R. M.

398 Dupont St.

Toronto

West, L. R. 323 Armstrong Block,
Edmonton, Alta. (RM)

\section{Affiliate Members}

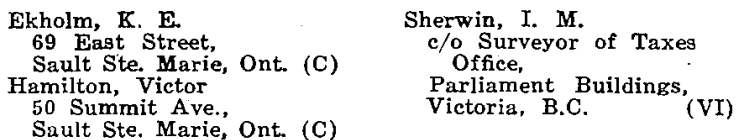

\section{NeW Student Members}

UNIVERSITY OF TORONTO

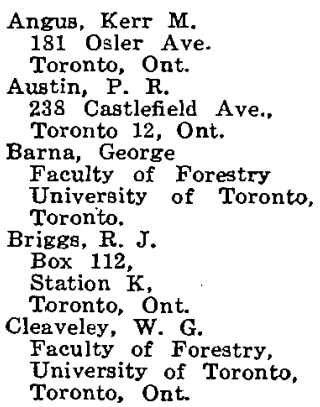

Deacoff, G. M.. 67 Raymond Ave,, Toronto 9, Ont.

Dowsett, Wm. Chas. 124 Glencairn Ave,
Toronto 12 , Ont.

Grant, William M. 159 Laclie St. Orillia, ont

Hoogan, Arnold $\mathrm{H}$. 478 Salem Ave. Toronito, Ont.

Hooke, Harry G. 4 Church St., Thornhill, Ont
Lambden, John R. Ŕd. 32 Marchmount

MeKay, John 171 Collier St., Toronto, Ont

Mepham, V. A. 270 Homewood Ave., Hamilton, Ont.

Morison, R. W. 210 Lawrence Ave. E. Toronto 12, Ont.

Mullen, J, L.

42 Mountain Ave. Hamilton, Ont. 